\title{
¿El retraso en el diagnóstico de la parálisis cerebral infantil genera mayores consecuencias en el desarrollo psicomotor?
}

\author{
Delay in the diagnosis of child cerebral paralysis generates major consequences in psychomotor \\ development?
}

\section{Sr. Editor:}

La parálisis cerebral (PC) describe a un grupo de alteraciones del desarrollo del movimiento y de la postura, causadas por alteraciones no progresivas del cerebro durante el desarrollo fetal o en la infancia, que provocan una limitación de la actividad. Las alteraciones motoras se acompañan con frecuencia de alteraciones sensitivas, cognitivas, de la comunicación, de la percepción y del comportamiento (1).

En la actualidad con los avances de la tecnología en nuestro país, los niños que tienen complicaciones durante el nacimiento tienen una rápida intervención del personal de salud, que hace que disminuya la mortalidad perinatal (2); en nuestra experiencia estos niños luego de ser estabilizados por lo general son dados de alta, no siendo detectados como niños de riesgo o con sospecha para una parálisis cerebral; algunos padres refieren que son ellos o un familiar cercano los que detectan el retraso en el desarrollo psicomotor.

El diagnóstico definitivo de la PC debe realizarse después del año de edad en los niños nacidos a término y a los 15 a 18 meses. si nació de forma prematura (3); sin embargo, para algunos autores el diagnostico se debe realizar a los 24 meses de edad, primordialmente en base a la evaluación del neurodesarrollo y el examen neurológico (4).
Los déficits neuropsicológicos asociados y la calidad de vida de los niños dependen principalmente de dos factores: el lugar anatómico de la lesión y el momento en el que se produce; lesiones en el hemisferio izquierdo repercuten en el lenguaje y lesiones en el hemisferio derecho en la atención y viso percepción, entonces la intervención temprana en niños con PC mejoraría la reorganización cerebral. Aisen citado por Vega et al. (5), propone que el momento de la lesión es importante debido a que mientras más temprana sea la lesión y en consecuencia el abordaje terapéutico, mejores serán los resultados debido a la neuroplasticidad.

La PC conlleva a alteraciones músculo esqueléticas y múltiples deformidades (6), que deberían tener una intervención adecuada. En su estudio Vila et al. (7), encontró en niños con PC que acudieron a consulta que solo el 39,5\% fueron diagnosticados antes de los dos años, la edad media al momento del diagnóstico fue $4,1 \pm 3$,2 años para el resto de los niños, concluyendo que el diagnostico fue tardío, el tiempo para esperar atención especializada fue prolongado, esto se explicaría por los diferentes factores en el manejo de nuestro sistema de salud y señala que el retraso en el diagnóstico de la parálisis cerebral debe ser una variable a incluirse en futuras investigaciones, para atender de manera adecuada a estos pacientes.

1 Departamento de Investigación, Docencia y Rehabilitación Integral en el Desarrollo Psicomotor; Instituto Nacional de Rehabilitación ‘Dra. Adriana Rebaza Flores’ Amistad Perú-Japón. Lima, Perú.

Licenciada Tecnólogo Medico, especialidad Terapia Física y Rehabilitación. 
Moraleda et al. (8), hallaron en su estudio de niños hasta los seis años que, debido a la condición dinámica del cerebro los niños con diagnóstico de parálisis cerebral pueden mejorar significativamente en sus capacidades personales, sociales, adaptativas y cognitivas y en la comunicación receptiva cuando reciben tratamiento continuo.

Existen dificultades en el sistema de salud de nuestro país que se deben tener en cuenta para la intervención temprana de manera integral, así como formular lineamientos de salud a nivel nacional orientadas a mejorar el bienestar y calidad de vida de los pacientes así también futuras investigaciones que evidencien las consecuencias de una atención a destiempo.

\section{Roxana Leonor Ruiz - Pingo ${ }^{1, \text { a. }}$}

\section{Correspondencia:}

Roxana Leonor Ruiz - Pingo

Correo electrónico: roxanr_2@hotmail.com

\section{REFERENCIAS BIBLIOGRÁFICAS}

1. López J, López L. Fisiología clínica del ejercicio. Madrid, España: Editorial Médica Panamericana S.A; 2008.

2. Instituto Nacional de Estadística e Informática. Perú Encuesta Demográfica y de Salud Familiar. Lima: Instituto Nacional de Estadística e Informática; 2017. p. 50. (citado en octubre 2018). Disponible en: https://www.inei.gob.pe/media/MenuRecursivo/ publicaciones_digitales/Est/Lib1525/index.html
3. Zitelli B, Davis H. Atlas de diagnóstico mediante exploración física en pediatría. Madrid, España: Elsevier; 2009.

4. Fajardo A. Detección temprana de las alteraciones en el neurodesarrollo. En: Garrido A. Guías de pediatría práctica basadas en la evidencia. 2a Edición. Bogotá: Editorial panamericana; 2009. p. 41.

5. Vega M, Ensenyat A, García-Molina A, AparicioLópez C, Roig-Rovira T. Déficits cognitivos y abordajes terapéuticos en parálisis cerebral infantil. Acción Psicológica. 2014; 11(1):107-117.

6. Koop S. Parálisis cerebral: Panorama general manejo de las deformidades de la columna vertebral. En: Born C. Ortopedia. $2^{\circ}$ ed. Buenos Aires: Editorial. Médica Panamericana S.A; 2004. p. 1734.

7. Vila J, Espinoza I, Guillény D, Samalvides F. Características de pacientes con parálisis cerebral atendidos en consulta externa de neuropediatría en un hospital peruano. Rev Perú Med Exp Salud Pública. 2016; 33(4):719-724.

8. Moraleda E, Romero M, Cayetano M. La parálisis cerebral como una condición dinámica del cerebro: un estudio secuencial del desarrollo de niños hasta los 6 años de edad. Univ Psychol. 2013; 12 (1):119-127. 